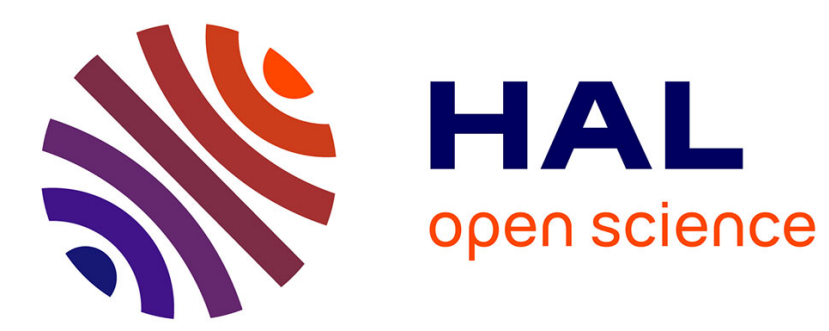

\title{
Justice de proximité et proximité de la justice. Les maisons de justice et du droit
}

Anne Wyvekens

\section{To cite this version:}

Anne Wyvekens. Justice de proximité et proximité de la justice. Les maisons de justice et du droit. Droit et Société, 1996, 33, pp.363-388. 10.3406/dreso.1996.1376 . halshs-02269930

\section{HAL Id: halshs-02269930 \\ https://shs.hal.science/halshs-02269930}

Submitted on 23 Aug 2019

HAL is a multi-disciplinary open access archive for the deposit and dissemination of scientific research documents, whether they are published or not. The documents may come from teaching and research institutions in France or abroad, or from public or private research centers.
L'archive ouverte pluridisciplinaire HAL, est destinée au dépôt et à la diffusion de documents scientifiques de niveau recherche, publiés ou non, émanant des établissements d'enseignement et de recherche français ou étrangers, des laboratoires publics ou privés. 
Justice de proximité et proximité de la justice. Les maisons de justice et du droit

Anne Wyvekens

\section{Citer ce document / Cite this document :}

Wyvekens Anne. Justice de proximité et proximité de la justice. Les maisons de justice et du droit. In: Droit et société, $n^{\circ} 33$, 1996. Les professionnels du divorce. pp. 363-388;

doi : https://doi.org/10.3406/dreso.1996.1376

https://www.persee.fr/doc/dreso_0769-3362_1996_num_33_1_1376

Fichier pdf généré le 15/05/2018 


\title{
Résumé
}

Sous des dehors très consensuels, la notion de " justice de proximité » recouvre aujourd'hui des contenus multiples. L'article se donne pour objectif de contribuer à en préciser la portée, à partir de l'analyse de l'activité des maisons de justice du Rhône. Qu'il s'agisse du traitement de la petite délinquance ou de l'accès au droit, l'observation des pratiques conduit à relativiser l'ampleur d'une proximité géographique. De même, la proximité humaine a tendance à s'effacer, au profit de l'avènement d'une proximité temporelle. Cet effet des préoccupations identitaires de l'institution judiciaire rencontre en réalité - et c'est ce qui apparaît intéressant - les attentes des différents acteurs locaux. Ceux-ci semblent en effet valoriser, au moins autant que la justice de proximité, justice différente venue traiter les problèmes spécifiques de certains quartiers, la proximité de la justice, rapprochement d'une justice identique à elle-même, avec ses conséquences sur le plan tant symbolique qu'instrumental.

\begin{abstract}
" Proximity Justice » and the Closeness of Justice. The " Houses of Justice and Law ».

Today, the apparent consensual notion of « proximity justice » (neighbourhood justice) covers many meanings. This paper aims to specify its scope, through the analysis of the activities of the " houses of justice » located in the Rhône department. The survey of the usages and practices of both the treatment of petty crime and access to law leads to the acknowledgement of the relatively limited scope of geographical proximity. In the same way, human proximity tends to give way to the rise of temporal proximity. Interestingly, the effect of the identity concerns of the judicial institution in fact meets the expectations of the various local actors. The latter seem to value, at least as much as " proximity justice » - a different type of justice which has been developed to deal with the specific problems of some neighbourhoods - the moving closer of a non-specific judicial institution faithful to its true nature, with its consequences on both the instrumental and symbolic level.
\end{abstract}




\section{Justice de proximité}

\section{et proximité de la justice. \\ Les maisons de justice et du droit}

\section{Anne Wyvekens *}

\section{Résumé}

Sous des dehors très consensuels, la notion de "justice de proximité » recouvre aujourd'hui des contenus multiples. L'article se donne pour objectif de contribuer à en préciser la portée, à partir de l'analyse de l'activité des maisons de justice du Rhône. Qu'il s'agisse du traitement de la petite délinquance ou de l'accès au droit, l'observation des pratiques conduit à relativiser l'ampleur d'une proximité géographique. De même, la proximité humaine a tendance à s'effacer, au profit de l'avènement d'une proximité temporelle. Cet effet des préoccupations identitaires de l'institution judiciaire rencontre en réalité - et c'est ce qui apparaît intéressant - les attentes des différents acteurs locaux. Ceux-ci semblent en effet valoriser, au moins autant que la justice de proximité, justice différente venue traiter les problèmes spécifiques de certains quartiers, la proximité de la justice, rapprochement d'une justice identique à elle-même, avec ses conséquences sur le plan tant symbolique qu'instrumental.

Justice de proximité - Médiation - Modernisation - Politique de la ville Territorialisation.

\section{Summary}

"Proximity Justice" and the Closeness of Justice. The "Houses of Justice and Law "

Today, the apparent consensual notion of « proximity justice » (neighbourhood justice) covers many meanings. This paper aims to specify its scope, through the analysis of the activities of the " houses of justice " located in the Rhône department. The survey of the usages and practices of both the treatment of petty crime and access to law leads to the acknowledgement of the relatively limited scope of geographical proximity. In the same way, human proximity tends to give way to the rise of temporal proximity. Interestingly, the effect of the identity concerns of the judicial institution in fact meets the expectations of the various local actors. The latter seem to value, at least as much as "proximity justice" - a different type of

\section{L'auteur}

Chargée de recherche au CNRS (URA 1267), chercheur associé à l'Équipe de recherche sur la politique criminelle, chargée d'enseignement à la faculté de droit de Montpellier. Après avoir centré ses travaux sur les transformations du système de justice pénale, elle s'intéresse plus particulièrement aux rapports que celui-ci entretient avec la politique de la ville, ainsi qu'à cette politique en tant que telle. Ces études ont donné lieu à plusieurs rapports de recherche, notamment pour le ministère de la Justice et la Délégation interministérielle à la ville.

*CEPEL/ERPC,

Université de Montpellier I, Faculté de droit, des sciences économiques et de gestion, 39 rue de l'Université, F-34060 Montpellier cedex. 


\section{A. Wyvekens \\ Justice de proximité et proximité de la justice. Les maisons de justice et du droit}

1. Le rapport sur les maisons de justice et du droit présenté au garde des Sceaux, en férrier 1995 , par le député $G$. Vignoble enseigne que sous cette appellation générique on trouve aujourd'hui, disséminées à travers la France, 32 "structures ». Soulignant qu'elles se caractérisent par la diversité de leur mode de fonctionnement et de financement, il décline un ensemble de propositions destinées à constituer un cadre de référence. Plusieurs monographies rendent compte de la diversité des pratiques : P.A. VID:I-NAQLET, L'action judiciaire dans la ville. L'exemple de l'antenne de justice de Gennevilliers, Narseille, CERPE, juin 1994 (cette " antenne de justice " figure au nombre des structures répertoriées par la Chancellerie comme " maisons de justice ") ; N. BRIAND, Les maisons de justice et de média. tion du ressort du TCI de SaintPierre, lle de la Réunion, Observatoire départemental de la Réunion, décembre 1993 ; S. MALIET, Les maisons de justice et du droit : image ou réponse d'une justice en crise ?, mémoire de DEA de théorie générale et philosophie du droit, Université Paris X Nanterre, 1992 (à propos des maisons de justice du Val d'Oise); A. WrVEKENS, Analyse de l'activité des maisons de justice et du droit du tribunal de grande instance de Lyon, Montpellier, ERPC/Ministère de la Justice/TGI de Lyon, 1995.

2. Au moment de la parution du rapport Vignoble (précité), le journal Le Monde titre, le 18 mars 1995 : « Le rapport Vignoble dresse un bilan positif des maisons de justice. Soulignant l'intérêt de la médiation pénale, le député du Nord estime cependant nécessaire de clarifier le financement et les statuts de ces structures judiciaires de proximité. »

3. J.P. Bonafi-SchnitT, La médiation : une justice douce, Paris, Syros, 1992 . justice which has been developed to deal with the specific problems of some neighbourhoods -, the moving closer of a non-specific judicial institution faithful to its true nature, with its consequences on both the instrumental and symbolic level.

Mediation - Modernization - Proximity justice - Territorialisation - Urban policy.

Interpellée dès le début des années quatre-vingt par les nouvelles politiques de prévention qui, face à l'explosion et à l'exploitation du sentiment d'insécurité, l'invitaient à participer, localement, à une approche partenariale des questions de délinquance, l'institution judiciaire y avait à l'origine difficilement trouvé sa place. Soucieuse de préserver sa spécificité dans une alliance problématique entre prévention et répression, craignant de voir entamée son indépendance face aux pouvoirs locaux, freinée par les contraintes matérielles et organisationnelles internes, sa participation aux instances locales de prévention était souvent restée limitée à l'engagement - au demeurant dynamique et inventif - de quelques individualités. La pression exercée par la politique de la ville suscitait en quelque sorte plus de questions - sur le rôle et le fonctionnement de l'institution - que de réponses.

Les questions initiales, et notamment celle, lancinante, du "que fait la justice? " face à la petite délinquance, restaient néanmoins posées, et des éléments de réponse - ancrage local, souci de restauration du lien social - prêts à être utilisés. C'est dans cette perspective que le modèle lyonnais de maisons de justice ${ }^{1}$ peut, dans son ensemble, être analysé. Le discours dominant construit volontiers aujourd'hui la représentation des maisons de justice autour de deux notions, celle de médiation d'une part et celle de proximité d'autre part ${ }^{2}$. Derrière cette image consensuclle, l'analyse des pratiques met en évidence une réalité moins homogène. Qu'il s'agisse de la "justice douce » ${ }^{3}$ ou de la « justice de proximité ", c'est une articulation complexe entre des logiques partiellement contradictoires qui se donne à voir. Au lieu d'entrer d'emblée dans le partenariat local, l'institution judiciaire a en effet élaboré sa propre réponse face aux priorités dégagées par la politique de la ville. Tout en visant à intégrer un souci de recréation du lien social, à partir d'une tentative d'inscription territoriale, les maisons de justice, créations de l'institution, en véhiculent dès lors la logique interne. Celle-ci, loin de demeurer inactive, entre en dialogue avec les préoccupations qu'introduit, de l'extéricur, la politique de la ville.

On n'abordera pas de front, ici, l'analyse de "la » médiation pratiquée dans les maisons de justice. On laissera entrevoir néanmoins, rejoignant les propos de J. Faget sur la récupération de la 
médiation pénale par l'institution judiciaire ${ }^{4}$, les distances que l'activité judiciaire des maisons de justice, profondément impré gnée de la logique de l'institution, prend par rapport au modele médiateur: bien que réellement centrée sur la rencontre entre le mis en cause et sa victime, la " troisieme voie " s'apparente plus souvent au classement sous condition, témoignant parfois même de résurgences d'une approche répressive ${ }^{j}$. Le présent propos, adoptant un point de vue moins centré sur le judiciaire, concernera la notion de "justice de proximité ». En quoi les maisons de justice contribuent-elles à donner corps à l'exigence de proximité mise en avant par la politique de la ville? Quel est le contenu de cette proximité?

\section{"Justice de proximité » : une formule fourre-tout?}

Le 2 octobre 1992, la Chancellerie fait paraître une circulaire relative aux "réponses à la délinquance urbaine " ${ }^{6}$. Parmi ces réponses figure notamment le souci de "rapprocher la justice du justiciable ». Le texte propose d'abord la création d'observatoires locaux de la délinquance. Il évoque ensuite « le développement d'une justice de proximité [devant] aussi contribuer à ce rapprochement ». Il s'agit ici, devant "l'incompréhension manifestée par la population de certains quartiers face à l'activité des services chargés de l'application de la loi », de créer " dans les sites urbains qui le nécessitent » des antennes de justice ou des maisons de justice, ainsi que de mieux utiliser les tribunaux d'instance. La troisième forme que devra prendre le rapprochement souhaité concerne, enfin, une meilleure explication de l'action de la justice. Trois brochures accompagnent la circulaire. L'une d'entre elles est intitulée La justice de proximité. Les maisons de justice et du droit. Introduite par une note d'orientation qui, sous le titre "Réaffirmer le droit dans la ville. La justice de proximité ", évoque les trois types d'expérimentations que sont la sectorisation des parquets, les antennes de justice et les maisons de justice, elle est ensuilc consacrée essentiellement à ces dernières.

En février 1994, les sénateurs H. Haenel et J. Arthuis remettent au garde des Sceaux un rapport intitulé Propositions pour une justice de proximité 7 . Le document, résultat des travaux d'une commission installée en septembre 1993, comprend trois parties: "Rapprocher les Français de leur justice », " Recentrer le juge sur ses missions ", et "Juger à proximité ». Ses propositions ouvrent un éventail très large, comprenant notamment la création au niveau du tribunal d'instance d'un juge (non professionnel) des contentieux de proximité, le développement du juge unique, la consécration du tribunal d'instance comme juridiction de droit commun, la généralisation du traitement en temps réel des affaires
4. J. FAGEI, " La médiation pénale : une dialectique de l'ordre et du désordre ",

Déviance el Société, vol. 17, n'3, 1993, p. 221-233; et "La double vie de la médiation ", Droit et Société, n'29, 1995, p. 25-38.

5. Cf. à ce sujet A. WrTtkess, "Le traitement de la délinquance urbaine en maison de justice ", Justices, n' 2, 1995, p. 91-101.

6. Circulaire NOR.Jl'S.D.92-300222 (C (RIM. 92.13/SDJC-2.1().92.

7. H. HAENEL et J. ARTHUIS, Propositions pour une justice de proximité, ronéo, s.d. 
A. Wyvekens

Justice de proximité et proximité de la justice. Les maisons de justice et du droit
8. G. VIGNOBLE, Les maisons de justice et du droit, op. cit.

9. « Justice concentrée, justice de proximité. Enjeux autour des modèles de justice ", Paris, École nationale de la magistrature, 15-16 décembre 1994.

10. Cf. également H. HAENEL, « Justice de proximité. Premier bilan ", pouvoirs, n' 74, 1995, p. 93-103.

11. Propositions pour une justice de proximité, op. cit., p. 2. pénales, le développement de la médiation pénale, la création d'un contrat de procédure en matière civile.

En février 1995, c'est au tour du député G. Vignoble de rendre son rapport, relatif aux maisons de justice et du droit ${ }^{8}$. " L'image négative et l'apparente inefficacité de l'institution judiciaire, y liton en préambule, remettent en cause l'identité même et les fondements de la justice. Les changements profonds dans l'évolution de notre société justifient, et il n'est plus nécessaire de le prouver, d'un besoin vital de justice de proximité, d'un rapprochement dans le temps et dans l'espace entre l'institution judiciaire et nos concitoyens. "

Ces quelques textes, tous points de repère plus ou moins officiels de la "justice de proximité", donnent une première idée, avant toute observation des pratiques, de la multiplicité des contenus que peut prendre la formule. La même impression se dégageait, il y a quelques mois, lors d'une session de formation organisée par l'École nationale de la magistrature sous le titre "Justice concentrée, justice de proximité ${ }^{9}$. De l'historien au sociologue, du magistrat à l'économiste, du Moyen Âge aux cahiers de doléances de 1789, de l'architecture judiciaire à la modernisation de l'institution, de l'arbitrage international au centre commercial Carrefour de Stain... autant d'interventions, autant de discours différents se glissant sans difficulté apparente dans le mëme moule. Sous les dehors consensuels dont la revêt l'air du temps, qu'elle soit envisagée en elle-même ou comme miroir d'une autre justice, la "justice de proximité " apparait comme une notion "fourretout ». La richesse entrevue appelle, afin de pouvoir être exploitée, une démarche de construction à laquelle on voudrait contribuer, à partir d'un terrain spécifique, celui des maisons de justice lyonnaises.

\section{La proximité : géographique, affective, temporelle}

Une première tentative, sinon de construction, du moins de classification, apparait dans le travail de la commission HaenelArthuis ${ }^{10}$. S'interrogeant sur " la notion même de proximité ", elle y voit trois dimensions: géographique d'abord, mais également affective et temporelle : «Le concept de proximité désigne d'abord bien évidemment ce qui est proche géographiquement mais cette proximité est aussi affective - l'expression "les proches" renvoie à l'idée de parenté -, c'est aussi ce qui est "imminent", ce qui va ou doit arriver, ce qui est rapproché dans le temps ${ }^{11}$. " Appliquées à l'institution judiciaire, ces trois acceptions conduisent, poursuit la commission - et l'on note au passage que l'ordre des proximités subrepticement se modifie - à parler d'une justice "plus familière, c'est-à-dire plus accessible, plus lisible, peut-être davantage à taille humaine; plus proche dans le temps, c'est-à-dire capable de 
résoudre de façon plus rapide les litiges qui lui sont soumis, et plus proche des justiciables sur le terrain $" 12$.

Proximité géographique, proximité affective, proximité lemporelle ; justice plus familière, plus proche dans le temps, plus proche sur le terrain... L'analyse des pratiques des maisons de justice et de leurs représentations, à la lumière notamment de cette ébauche de grille à trois entrées, fait voir que la proximité annoncée, relativement limitée là où on l'attend, existe pourtant, mais sous d'autres formes ou concernant d'autres objets que ceux qui figurent dans le discours dominant. On montrera dans un premier temps comment les maisons de justice du Rhône ne constituent pas une décentralisation de l'institution judiciaire, venue traiter de manière spécifique les problèmes spécifiques de certains quartiers (I). On examinera ensuite, abandonnant le point de vue interne à l'institution pour partir de celui de ses partenaires locaux, en quoi les maisons de justice contribuent néanmoins à un "rapprochement " de la justice et comment celui-ci peut être analysé (II).

\section{Maisons de justice et justice de proximité}

Les maisons de justice concrétisent à première vue une proximité principalement géographique. Les évoquer renvoie en effet assez irrésistiblement à la lecture topologique de la question sociale ${ }^{13}$ qu'a progressivement instaurée la politique de la ville. Construite sur une approche locale, la politique de la ville raisonne en termes de zones, de quartiers, de territoires. Parallèlement à la notion de relégation ${ }^{14}$, notion clé de la problématique des banlieues, a émergé celle de "quartier hors droit ". Plus essentiellement que des espaces urbains où la police ne pénétrerait plus qu'à contrecœur, ces quartiers sont décrits comme des quartiers « où la référence à la norme étatique ou sociale a perdu ou est en train de perdre de sa consistance " 15 : à côté de la banalisation des actes de vandalisme ou de violence, on y constate, chez des habitants se trouvant souvent en position d'assistés, une perception de la règle de droit comme étant d'abord d'application individuelle. En résulte une pression constante pour négocier la règle, qui est dès lors rarement prise en compte comme une norme au sens fort, dans sa dimension collective. Parallèlement, dans ces quartiers, les principaux services publics pouvant jouer le rôle de référence à la loi se situent en position de retrait: police hésitante, justice lointaine, services sociaux fermant leurs antennes ou diminuant leurs effectifs.

Dans ce contexte, la " justice de proximité » aurait pour vocation de réintroduire le droit dans les territoires qu'il a désertés. Le rapport Vignoble annonce ainsi : "Pour garder sa crédibilité et préserver sa mission première qui est le maintien de la paix sociale par l'exercice de l'action publique, il apparait désormais évident
12. Ibid., p. 3.

13. J. DONZELOT et M.-C. JAILLET (dir.), Les zones urbaines défavorisées. Leurs diagnostics, les politiques en leur direction et la question de la justice sociale en Europe et en Amérique du Nord, séminaire international (Plan urbain/OTAN), compte rendu des séances, vol. I, printemps 1995, p. 4-9.

14. J.-M. DEL ARUE, Banlieues en difficultés : la relégation, Paris, Syros, 1991.

15. À titre d'illustration de la notion de quartier hors droit, voir par exemple C. Mével. et A. WYYFEFEN, Diagnostic local de sécurité. Ville d'Avignon, 2 partie, Paris, ARIS, 1992 (ronéo), p. 33 et suiv. 
A. Wyvekens Justice de proximité et proximité de la justice. Les maisons de justice et $d u$ droit
16. G. Vignoble, I.es maisons de justice et du droit, op. cit., p. 4. 17. Développement social des quartiers.

18. G. VIGNobI.F, Les maisons de justice et du droit, op. cit., p. (6. que l'institution judiciaire soit dans l'obligation de faire un pas vers le citoyen. Partant de ce constat et devant l'existence dans certains quartiers "difficiles" de zones de "non-droit", où l'idée même de justice et de droit a complètement disparu, l'institution judiciaire a ćté conduite dès 1990, sous l'impulsion d'un procureur de la République, à mettre en ceuvre une démarche nouvelle au sein même de ces quartiers. Les maisons de justice et du droit sont nées, "assises immobilières de la justice", ayant la double vocation de rétablir et favoriser l'accès au droit et de concourir sur place all traitement de la petite délinquance ${ }^{16}$."

Cette figure est celle d'une justice venue s'installer dans des lieux spécifiques, afin d'y développer un mode d'action spécifique. Or, qu'il s'agisse du traitement de la petite délinquance (I.1) ou de l'accès au droit (1.2), l'observation des pratiques conduit à relativiser l'ampleur de l'inscription territoriale des maisons de justice.

\section{I.1. Le traitement de la petite délinquance : une « action judiciaire pénale différenciée et décentralisée »?}

Le rapport Vignoble, après avoir souligné la diversité qui caractérise aujourd'hui les modes de fonctionnement des structures rassemblées sous l'appellation "maisons de justice", rappelle qu'« à l'origine il s'agissait d'assurer dans des zones classées DSQ ${ }^{17}$ une présence judiciaire spécifique proche des justiciables, par l'institution de lieux d'exercice de nouveaux modes de traitement des procédures pénales " 18 . À quartiers spécifiques, justice spécifique? La complexité de la "proximité » qu'instaurent les maisons de justice se lit à plusieurs niveaux, qui se superposent partiellement. On tentera de les démêler et d'apprécier les enjeux de cette "proximité » en montrant successivement les limites de la proximité géographique et de la proximité humaine, au profit de l'avènement d'une proximité temporelle.

\section{a. Une proximité géographique relative}

Les conventions instituant les maisons de justice lyonnaises, qui en présentent la mission judiciaire comme " une action judiciaire pénale différenciée et décentralisée », précisent ensuite en quoi cette action se veut décentralisée. "La localisation de la Maison de la justice et du droit au centre-ville |près du centre-ville] lui permettra de bénéficier d'un effet de proximité. Elle a pour vocation de connaitre: des infractions dont l'auteur réside à /ville d'implantation|, des infractions commises à $\mid . .$. , des infractions commises dans le Rhône et dont la victime réside à |.... Elle est saisie de ces infractions: soit par les services d'enquête habituels (police, gendarmerie), soit par les avocats, soit par les victimes, soit 
par les différentes institutions 19." Ia localisation de la structure, la provenance des infractions traitées et le mode de saisine apparaissent comme les trois points d'ancrage d'un effet de proximite géographique.

S'agissant de la localisation, le texte même de la convention, prenant acte des choix opérés sur le terrain, reflète le premier infléchissement apporté au programme initial: trois des quatre maisons de justice du Rhône ne se situent pas au coxur des quartiers les plus difficiles ${ }^{20}$. Si les villes d'implantation comportent des quartiers dégradés, visés par la politique de la ville, le choix du centre-ville - décision relevant de la compétence des municipalités, qui fournissent les locaux - n'en est pas moins un choix délibéré. Deux arguments sont mis en avant par les élus ou fonctionnaires municipaux : le service de l'ensemble de la population, d'une part, le souci de ne pas stigmatiser davantage des quartiers qui le sont déjà, d'autre part. Seule la maison de justice de Vaulx-en-Velin se trouve implantée, selon un choix tout aussi délibéré, dans la ZUP. "Le concept, tel qu'il a été reçu ici, présuppose que la maison de justice est pour la ZUP. De toute façon, la règle ici, c'est de réinstaller les activités publiques dans la ZUP. " On reviendra sur les enseignements qui peuvent être tirés de ce premier constat.

Se cumulant aux décisions prises par les villes en matière d'implantation, la manière dont l'institution judiciaire organise le traitement des affaires pénales en maison de justice, qu'il s'agisse du rattachement géographique des infractions traitées ou du mode de saisine, contribue à relativiser l'importance accordée à des objectifs de proximité territoriale.

Si l'activité judiciaire des maisons de justice se dit décentralisée, c'est en effet sur fond et en tant qu'élément d'un dispositif parquetier d'ensemble qui trouve sa place, lui, au palais de justice: le "traitement direct " des affaires pénales. Instauré en même temps que les maisons de justice, ce dispositif consiste à réscrver aux affaires élucidées, plus précisément les affaires pour lesquelles une personne mise en cause se trouve dans les locaux de la police ou de la gendarmerie, un traitement " en temps réel ». Les officiers de police judiciaire sont tenus de prendre contact téléphoniquement avec la Section de traitement direct (STD), afin que le magistrat du parquet puisse, sur leur compte rendu et immédiatement, donner à l'affaire l'orientation qui lui paraîtra la plus adéquate : instruction, citation directe, convocation par officier de police judiciaire ou convocation en maison de justice. Contrairement au système antérieur, dans lequel seules les affaires graves, susceptibles de nécessiter un défèrement au parquet, faisaient l'objet d'un appel à la permanence, les autres étant transmises au parquet par courricr ct traitées "en différé », une orientation immédiate est
Droit et Sociéte 3.3-1996;

19. Article At de la convention.

20. Les maisons de justice du ressort du TGI de Lyon sont au nombre de quatre : Bron, Lyon 8: Villeurbanne et Vaulx-enVelin. La convention de Vaulx en-Velin indique " près du centre-ville "; les trois autres, " au centre-ville". Le cas de la maison de justice de Lyon est un peu plus complexe, dans la mesure où il peut se décliner à deux niveaux. Présentée comme la maison de justice de l'ensemble de la ville de Lyon, son implantation dans le 8 arrondissement la situe effectivement dans un arrondissement peu favorisé. À l'intérieur même de cet arrondissement, toutefois, la maison de justice a été implantée non pas dans les zones d'immeubles, mais à côté de la mairie d'arrondissement et du commissariat - la rapprochant ainsi des choix opérés à Bron et à Villeurbanne. Les conventions relatives aux maisons de justice du Val d'Oise, créées peu de temps avant celles du Rhône par le même magistrat et sur un modèle analogue, situent cellesci « au cocur d'un quartier difficile ». Selon des informations récemment recueillies à Pontoise, la maison de justice de Cergy est en passe de quitter son actuelle zone d'implantation (Z.VP pavillonnaire) pour s'installer en centre-ville. 


\section{A. Wyvekens}

Justice de proximité et proximité de la justice. Les maisons de justice et du droit

21. Un dépouillement de dossiers traités dans les maisons de justice du Rhône montre le caractère limité de la proximité (au sens d'existence de l'un au moins des trois critères de rattachement prévus par la convention) :

Bron : $21,57 \%$;

Vaulx-en-Velin : $20,41 \%$; Villeurbanne : 49,49\%; Lyon $8 ": 18,75 \%$.

22. Dans ce sens, à propos de la médiation en général, voir C. DOURLENS et P.A. VIDAlNAQUET, L'autorité comme prestation. La police et la justice dans la politique de la ville, Marseille, CERPE, 1993. désormais donnée à un certain nombre d'affaires de petite et moyenne délinquance. Dans ce dispositif, les maisons de justice apparaissent comme une voie nouvelle dans un sens quantitatif autant que qualitatif. Si une différence qualitative de traitement s'impose en raison de la faible gravité de certaines affaires, les magistrats du parquet de Lyon reconnaissent sans états d'âme - et revendiquent - la mise en ceuvre d'une logique quantitative : le traitement direct n'est concevable que si une filière autre que l'audience correctionnelle permet d'absorber la masse d'affaires supplémentaires à traiter.

L'insertion de l'activité judiciaire des maisons de justice dans ce type de gestion globale du contentieux pénal n'est pas sans effet sur sa dimension territoriale. D'une part, on constate que les affaires traitées par les maisons de justice sont loin de provenir en priorité de leur lieu d'implantation : vu l'application centralisée du traitement direct, les maisons de justice, tout en étant situées dans quatre communes de l'est lyonnais, sont compétentes pour traiter des affaires de l'ensemble du ressort du tribunal de Lyon. Outre des informations chiffrées assez éloquentes ${ }^{21}$, l'enquête de terrain en témoigne avec cette information, pour le moins paradoxale, selon laquelle un certain nombre de rendez-vous donnés à des mineurs dans le cadre du "suivi " d'une médiation le sont dans les services du tribunal, plus proches de leur domicile que la maison de justice où ils avaient été convoqués. D’autre part, s'agissant des aspects qualitatifs du dispositif, les choix d'orientation excluent a priori de la compétence des maisons de justice la délinquance des quartiers les plus difficiles. La " troisième voie ", celle de la médiation pénale, est d'abord destinée à la résolution de petits conflits à propos desquels les poursuites traditionnelles paraissent excessives et qui, en l'absence de maison de justice, auraient fait l'objet d'un classement sans suite pur et simple. Au-delà de la localisation des maisons de justice et de leur compétence territoriale, le postulat d'une présence judiciaire de nature spécifique pour les quartiers hors droit apparaît de la sorte comme une contradiction dans les termes: quelle adéquation peut-il y avoir entre le traitement par la médiation de "petites affaires " et l'insécurité de certains quartiers 22 ? Les représentations des différents acteurs locaux, à nouveau, en attestent. Ainsi ce commissaire, déclarant qu'il «voit mieux la maison de justice pour les affaires de famille et de couple que pour les problèmes qui se posent dans des quartiers chauds ».

Les possibilités de "saisine locale» des maisons de justice, prévues par les conventions et sur lesquelles insistent également les magistrats du parquet, viennent-elles compenser les effets centralisateurs du traitement direct ? L'importance que cet objectif semble avoir est significative de la prégnance, au moins sur le plan symbolique, de la territorialisation. Mais, sur le terrain, sa réalisa- 

trice.

La saisine par les victimes elles-mêmes, ou par les protagonistes d'un conflit, n'apparaît jamais en tant que telle dans les procédures. Si quelques affaires sont traitées à la suite d'une demande locale, elles ont au préalable nécessairement réintégré le circuit traditionnel, centralisé, de la STD. À cet égard, le parquet précise avec force que développer la saisine locale ne peut en rien signifier un glissement vers la médiation sociale. Lc fondement de l'intervention est et demeure l'infraction. La nature de l'intervention ne se conçoit pas, du côté du parquet, autre que judiciaire.

Quant aux saisines par "les institutions", elles peuvent être de deux ordres. L'opération consiste, dans un cas comme dans l'autre, à court-circuiter la procédure traditionnelle afin de faciliter la saisine de l'institution judiciaire - en l'occurrence la maison de justice - dans des cas où les usagers sont perçus comme n'osant pas porter plainte, redoutant les conséquences de leur intervention. Il s'agit, d'une part, du « signalement " par les enseignants à la maison de justice de situations survenues à l'intérieur des établissements. L'objectif poursuivi consiste à tenter de réintroduire le droit au sein d'un milieu perçu par les magistrats comme " hors la loi ", dans la mesure où les différends, même graves, y sont abordés le plus souvent en circuit fermé. Il a d'autre part été question de développer une saisine de la maison de justice à partir des mains courantes des commissariats. L'argument est identique : si certains usagers répugnent à porter plainte - l'exemple couramment cité est celui des violences conjugales -, leur différend doitil pour autant rester sans réponse ? Pourquoi ne pas faire intervenir la «troisième voie "? Le développement de ces deux types de saisine reste toutefois marginal ${ }^{23}$. Outre les réticences apparaissant tant chez les enseignants que chez les policiers, ils font l'objet d'un débat de fond, qui revient à poser la question des limites de l'intervention pénale : si un certain nombre de "mains courantes " représentent des situations où les victimes redoutent des représailles, d'autres au contraire correspondent à l'utilisation rationalisée, par les victimes, d'une démarche dont elles savent pertinemment qu'elle n'aura pas de conséquences judiciaires. L'institution a-t-elle à leur forcer la main 24 ? S'agissant des enseignants, doitelle, dans le même objectif de facilitation de l'accès à la justice, réserver un traitement particulier à certains justiciables?

\section{b. Une proximité humaine restreinte}

Si donc la proximité géographique de l'action judiciaire des maisons de justice est réduite, au sens où il ne s'agit pas de traiter dans les quartiers, ni d'une façon adaptée à leur problématique, la délinquance des quartiers, une certaine proximité n'est toutefois

23. Seule une maison de justice sur les quatre a pratiqué une démarche volontariste importante dans ce sens.

24. C'est ainsi qu'une expérience d'exploitation des mains courantes, en 1992, se serait soldée par l'absence d'une partie des personnes concernées... et l'indignation des autres, considérant l'initiative du parquet comme abusive. 
A. Wyvekens

Justice de proximité et proximité de la justice. Les maisons de justice et du droit

25. I. THĖRY, Le démariage, Paris, Odile Jacob, 1993, p. 164. Le texte renvoie à J. Coms.sll..., "Les formes de justice comme mode de régulation de la famille, questions sociologiques posées par les tribunaux de famille sous la Révolution française ", in I. THÉRY et Ch. BIEr (dir.), La famille, la loi, l'ltat, Paris, Centre Georges Pompidou/Imprimerie nationale, 1989 , p. 274 et suiv. 26. Cf. notamment $\mathrm{Ph}$. Coppexs, "Médiation et philosophie du droit ", Archives de politique criminelle, n' 13, 1991, p. 13-23; C. LAZERGES, "Typologie des procédures de médiation penale ", in Melanges offerts it André Colomer, Paris, Litec, 1993, p. $217-234$; J. FACET, « la médiation pénale... », op. cit. ; E. LE ROY, "La médiation mode d'emploi », Droit et Société, n' 29, 1995, p. 39-55.

27. J. CARBONNIER, « Réflexion sur la médiation ", in I.a médiation: un mode alternatif de résolution des litiges? Lausanne, 14 et 15 nov. 1991, Zürich, Schulthess Polygraphischer Verlag, 1992, p. 13.

28. Grâce à l'intervention d'un travailleur social qui assurera, après le passage devant le magistrat, un "suivi " plus ou moins long, dans $30 \%$ environ des affaires traitées en maison de justice.

29. Ainsi, au cours de l'annce 1993, compte-ton à Lyon 3796 "médiations " pour 10132 jugements correctionnels (source : Ministère de la Justice, I)ACG, Sous-direction de la législation criminelle, Bureau des etudes, ictivité judiciaire 1993). pas absente de la démarche. C'est le contenu originel de la notion de "justice de proximité » qui réapparaît ici. Avant le développement des politiques territorialisées, et sans que l'expression connaisse alors la vogue qui est la sienne aujourd'hui, la " justice de proximité ", désignant notamment la pratique des juges des enfants, renvoyait en effet à une certaine conception, globale, de la fonction de justice : une justice qui, "privilégiant l'arbitrage et la négociation, est liée à une certaine représentation des rapports privé-public dans laquelle l'État n'est que faiblement interventionniste, et l'imaginaire social privilégie l'aspiration à une société communautaire sur l'ordre social imposé » 25. Proximité "humaine ", ou " affective ", dit-on à présent.

À défaut d'être proche d'un territoire, la justice des maisons de justice se veut proche des justiciables: inspirée des pratiques de médiation pénale, elle met en présence l'auteur d'une infraction et sa victime, afin de tenter de les faire parvenir à un accord réglant le différend qui les oppose. Ce conflit devient l'élément central de la logique qui gouverne la procédure, prenant la place de l'infraction, qui n'en apparaît plus que comme le symptôme. La proximité humaine est celle d'un mode de régulation se voulant centré plus sur la restauration du lien social que sur l'autoritarisme classique du pénal, celle d'une justice rendue dans un cadre non solennel, qui "prend son temps", pour renouer plutôt que pour trancher, le procès d'un homme faisant place au débat autour d'une relation. Il est frappant, à cet égard, d'observer comment le discours des acteurs se construit autour de la comparaison différentielle - avec l'audience correctionnelle.

Plusieurs éléments contribuent toutefois à relativiser cette " différence ». Le premier tient aux acteurs du traitement nouveau : confiée aux magistrats du parquet, la " médiation " des maisons de justice s'apparente a priori plus au classement sous condition qu'à une médiation véritable ${ }^{26}$. L'observation de la pratique montre en outre comment les "réflexes de magistrat " sont prompts à réapparaitre, comment les magistrats honoraires auxquels l'essentiel de cette activité est de fait délégué conservent " des sédiments de mentalité judiciaire » 27 , et comment sé réalise ainsi, plus ou moins consciemment, une véritable résurgence du pénal. L'analyse du contentieux traité va dans le même sens. Lne approche chiffrée fait en effet apparaitre le caractère minoritaire de ces conflits familiaux ou de voisinage si exemplaires de la logique de la médiation et si volontiers cités en exemple par les magistrats lyonnais. Si ces conflits-là bénéficient réellement d'un traitement plus humain ${ }^{28}$, le quotidien des maisons de justice - et il convient de signaler ici que sur le plan quantitatif leur activité est considérable 29 - est néanmoins fait d'un ensemble d'infractions - vols, dégradations, chèues sans provision ou abus de confiance, violences, délits de fuite - dont la caractéristique commune essentielle, outre qu'elles 
ne sont pas contestés, est moins l'inscription d'un conflit dans le temps que l'existence d'un préjudice susceptible d'être réparé. Enfin, l'inscription de cette "médiation " dans le dispositif du traitement direct fait se rencontrer deux temporalites qui apparaissent difficilement conciliables: jusqu'à quel point le "temps réel» permet-il à la justice de "prendre son temps "?

\section{c. L'avènement d'une proximité temporelle}

La « justice de proximité » ne serait-elle alors, d'abord, qu'une justice rapide ${ }^{30}$ ? Une grande part de l'originalité du traitement de certaines affaires pénales en maison de justice se situe sans conteste au niveau de la rapidité et de l'efficacité, au moins symbolique, d'une réponse autrefois introuvable. Grâce au traitement direct, les infractions de petite et moyenne délinquance sont traitées dans un délai très bref : deux mois en moyenne s'écoulent entre les faits et la comparution en maison de justice. Pour les victimes, l'impression d'être prises en compte vient remplacer la frustration du classement sans suite ou de la convocation tardive. Les auteurs, eux, sont plus souvent présents et susceptibles d'être responsabilisés par la rencontre avec leur victime. D'autre part, et dans la mesure où il s'agit d'un dispositif global, on ne saurait négliger un autre effet du traitement direct, observable à l'échelon du tribunal: la multiplication des convocations par officier de police judiciaire. Le fait que la décision d'orientation soit prise à un moment où la personne mise en cause n'a pas encore quitté les locaux de garde à vue permet en effet d'avoir recours à cette procédure, au terme de laquelle, en outre, le jugement rendu sera réputé contradictoire. D'un côté comme de l'autre, c'est bien plutôt l'existence d'un traitement, et son caractère immédiat, que son adaptation à un contexte local qui prédomine. Face à la crise urbaine, cette forme de "justice de proximité " consiste moins à répondre ailleurs et autrement qu'à répondre et répondre vite à des situations autrefois demeurées sans réponse.

\subsection{L'accès au droit : une autre justice de proximité ?}

Ayant pris acte du caractère peu décentralisé de l'activité judiciaire des maisons de justice, l'observateur se console à la perspective d'une analyse de leur activité juridique. L'activité juridique des maisons de justice n'est-elle pas le nœud de la construction d'une identité locale de la structure ? Restaurer le droit dans les cités, ce n'est pas seulement réintroduire le droit comme référence, c'est également fournir à leurs habitants l'accès à l'information juridique, rendre plus proche un droit considéré ici comme ressource pour les usagers. Que !'on relise les conventions instituantes ou 
A. Wyvekens

Justice de proximité et proximité de la justice. Les maisons de justice et du droit

31. G. VIGNOBLE, Les maisons de justice et du droit, op. cit., p. 6 .

32. Le terme " activité juridique " est impropre pour qualifier les permanences du comité de probation, dont la mission est de nature judiciaire. Nous l'utilisons ici au sens global de l'ensemble des activités autres que le traitement par le parquet d'un certain nombre de faits de petite délinquance.

33. Le cadre de l'étude menée à Lyon ne permettait pas de procéder à une analyse des représentations des usagers eux. mêmes.

34. "La maison de justice, c'est une maison un peu vide où il y a des interventions. On est de la maison sans en être. " (Entretien, travailleur social) entende les différentes déclarations d'intention, cet objectif fait partie intégrante des missions assignées aux maisons de justice. Ne les appelle-t-on pas, d'ailleurs, maisons de justice et du droit?

Malgré ce que cette présentation suggère d'équilibre entre les deux fonctions, le discours sur les maisons de justice - qu'il s'agisse du discours médiatique ou de celui des différents acteurs - accorde une place prépondérante à leur activité judiciaire. Une observation approfondie de la réalité du terrain confirme le caractère secondaire de leur activité juridique et de l'identité locale susceptible de s'y alimenter. Un certain nombre de signes existent, néanmoins, de la manière dont l'accès au droit pourrait engendrer une inscription plus marquée de la maison de justice dans la vie locale. Mais cette inscription territoriale reste contenue, à Lyon, dans des limites assez étroites.

\section{a. Une activité seconde et secondaire}

L'activité juridique et d'accueil des maisons de justice est historiquement seconde par rapport à leur activité judiciaire. Comme le rappelle le rapport Vignoble, " aux objectifs initiaux sont venues se greffer d'autres préoccupations s'inscrivant dans le développement d'une justice de proximité » 31 . Parmi ces préoccupations, on trouve à Lyon l'accès au droit et l'aide aux victimes, assurés respectivement par le barreau et par deux associations d'aide aux victimes, ainsi que les permanences décentralisées du comité de probation.

Si, ainsi qu'on l'a montré, l'activité judiciaire des maisons de justice ne s'avère pas nettement décentralisée, leur activité juridique ${ }^{32}$ n'apparaît ni à l'origine ni à l'usage constitutive d'un véritable noyau de proximité. Outre la question de l'implantation, déjà évoquée, la remarque se vérifie d'abord du côté des usagers. Selon les différents acteurs institutionnels interrogés 33 , même si les usagers ont de la maison de justice une représentation dans laquelle l'activité d'accès au droit est centrale, ils n'en ont qu'une connaissance réduite, et «n'ont pas le réflexe » de s'adresser à elle lorsqu'ils se trouvent confrontés à des difficultés pouvant mettre en jeu le juridique. Quant aux professionnels impliqués dans les différents aspects de l'activité d'accès au droit, le constat va dans le même sens: l'ensemble de ces acteurs et de ces activités n'apparaît pas constitutif d'une identité locale forte. C'est ainsi que parmi les acteurs interrogés, nombreux sont ceux qui déclarent se vivre comme des "invités" dans une maison qui n'est pas la leur ${ }^{34}$. Ils décrivent leurs relations avec les autres partenaires comme relevant plus du voisinage que de la coopération. Les interactions, d'ailleurs, sont lentes à se construire, tant il apparaît qu'elles dépendent d'initiatives individuelles plus qu'elles ne sont favorisées par la structure elle-même. Curicusement, les avocats ou 
permanents des services d'aide aux victimes, pourtant rencontrés afin d'être interrogés sur leur activité propre, ont tous manifesté une tendance lourde à parler abondamment de l'activité judiciaire de la maison de justice. On notera enfin que les permanences des uns et des autres occupent dans la grille horaire des maisons de justice une place sensiblement moins importante que celle réservée aux « médiations ».

En amont de la pratique quotidienne de la maison de justice, la manière dont les objectifs initiaux de cette activité juridique ont été définis témoigne d'un souci de l'inscrire assez étroitement à l'intérieur d'un champ qui soit essentiellement pénal et non social. Deux publics sont en effet visés, selon le texte des conventions: d'une part, les victimes d'infractions, d'autre part « le public et particulièrement les plus démunis ». Sans qu'on accorde une importance excessive à la forme, il n'est sans doute pas indifférent de noter que les victimes précèdent "le public ", ce public des quartiers, pourtant particulièrement "en manque " d'informations juridiques. S'agissant du contenu même de l'information juridique, d'autre part, même si un débat se développe actuellement sur ce point, il était convenu au départ qu'il s'agirait exclusivement d'informations relatives au droit pénal. Seconde dans le temps, l'activité juridique des maisons de justice reste secondaire par rapport au judiciaire et plus généralement par rapport au pénal, central.

\section{b. Une maison du droit : oui, mais...}

Si le développement local d'une activité autre que l'activité judiciaire ne semble pas constituer, pour le parquet, un objectif prioritaire, l'observation des différences apparaissant entre les quatre maisons de justice lyonnaises ${ }^{35}$ montre à la fois la possibilité d'un plus grand ancrage local, ses conditions et ses limites. L'exemple de Villeurbanne et celui de Vaulx-en-Velin illustrent cette question, sous deux angles différents, en mettant en évidence le caractère crucial de la position occupée par les acteurs directs ou indirects de l'accès au droit : service d'aide aux victimes, dans le premier cas, permanent et secrétaire dans le second. Dans un cas comme dans l'autre, le développement de cette activité, initié en dehors de l'action de l'institution elle-même, demeure relativement soumis à son approbation.

La maison de justice de Villeurbanne draine une quantité de demandes considérablement plus importante que les trois autres. La taille de la commune et une position centrale rendant sa maison de justice plus accessible que les autres à l'ensemble de la population lyonnaise ne sont pas la seule explication de cette affluence. Contrastant avec la présence réduite de l'aide aux victimes dans les
35. Vu le caractère centralisé de l'activité judiciaire, ces différences se situent essentiellement au niveau de l'activité juridique. 
A. Wyvekens

Justice de proximité et proximité de la justice. Les maisons de justice et du droit

36. Quatre demi-journées par semaine (et une affluence impressionnante), pour une petite demi-journée dans les trois autres maisons de justice. trois autres maisons de justice, Villeurbanne déploie en effet dans ce domaine une activité considérable ${ }^{36}$. Cette situation renvoie à la "personnalité » respective des deux associations concernées. Alors que Villeurbanne héberge un service d'aide aux victimes bien identifié, qualifié de performant, qui était solidement implanté localement avant sa participation à la maison de justice, la position du service intervenant dans les trois autres maisons de justice s'avère plus fragile. L'image des prestations proposées par les deux services reflète leur image globale. L'importante fréquentation locale que connaît la maison de justice de Villeurbanne peut dès lors très largement être attribuée au fait qu'elle abrite les permanences d'une association dont l'identité est particulièrement forte. La maison de justice s'est en quelque sorte contentée de récolter les fruits d'une position existante, qu'elle a ensuite contribué à consolider et dont elle a par ailleurs tiré profit pour structurer sa propre image locale. Dans les trois autres sites, en revanche, on s'aperçoit que l'association d'aide aux victimes, ne bénéficiant pas au départ d'une identité bien précise, n'a pas non plus bénéficié d'un apport d'image qui lui serait venu, en sens inverse, de la maison de justice. Cette remarque ne renvoie pas seulement au constat de la relativement lente constitution de l'identité locale de la maison de justice. Elle interroge sur sa possibilité réelle : jusqu'à quel point l'institution judiciaire, initiatrice de la structure, souhaite-t-elle le développement d'une telle identité, plus juridique que judiciaire, et surtout plus locale que centrale? La tension perceptible, à Villeurbanne, entre les deux partenaires forts que sont le parquet et l'association d'aide aux victimes prolonge la question : jusqu'à quel point l'institution supporte-t-elle de partager son emprise sur l'ensemble de la structure?

L'exemple de Vaulx-en-Velin illustre de manière plus explicite le même genre d'interrogation. Pour renvoyer aux avocats et services d'aide aux victimes, acteurs centraux de l'accès au droit, le permanent et le secrétaire des maisons de justice occupent une position parfois qualifiée de stratégique. Si leur intervention peut ne pas dépasser la simple orientation, ils se trouvent toutefois en situation d'assumer eux-mêmes une partie de l'activité d'information. Cette posture plus active semble volontiers adoptée à Vaulxen-Velin, où le permanent et la secrétaire, soucieux de faire de la maison de justice un lieu ressource, d'information immédiate, hésitent à renvoyer les habitants à un rendez-vous ultéricur avec des intervenants dont ils disent en outre avoir du mal à distinguer la spécificité. L'identité locale de la maison de justice se construirait donc, ici, autour de l'activité de conseil développée en continu par ses permanents. Acteurs qui ne sont pas totalement extérieurs à l'institution, tout en ne représentant pas uniquement ce que l'on pourrait appeler son "noyau dur », le judiciaire, leur situation, à la 
charnière entre le judiciaire et le juridique, met en évidence la ten-

sion susceptible de se créer entre ces deux pôles. C'est un peu comme si, en effet, l'un ne pouvait se développer qu'en menaçan! l'autre, comme si une concurrence, nécessairement, s'instaurait entre eux. Face à un modèle de maison de justice où le judiciaire occupe une place centrale, et où les acteurs de l'accès au droit font figure d'invités dans une maison qui n'est et ne deviendra pas la leur, la pratique observée à Vaulx-en-Velin donne à voir, sans le rédiser réellement, ce que pourrait être un autre modèle de maison de justice. La fonction judiciaire y serait considérée comme seconde. Au-delà de l'information juridique, c'est une véritable fonction sociale qui apparaitrait essentielle, le permanent et le secrétaire se vivant d'abord comme acteurs au sein du quartier, pour la population locale, à laquelle ils veulent répondre dans l'immédiat, avant d'être au service du judiciaire délocalisé. "Une approche plus liée à l'accueil qu'au déplacement du procureur sur les lieux... Faire de la maison de justice un lieu où on règle tout. " La médiation pourrait continuer d'y occuper une place importante, mais qui serait alors celle d'une médiation plus sociale que judiciaire.

La pratique lyonnaise, même à Vaulx-en-Velin, demeure celle du premier modèle. La tendance à faire de la maison de la justice " un lieu où on règle tout " ne s'est pas imposée. Les intervenants extérieurs y sont vécus par le parquet plus comme des invités que comme des partenaires. Les permanents et secrétaires accordent, bon gré mal gré, la priorité à leurs activités d'assistance au judiciaire. Les élus eux-mêmes, à Vaulx, sont intervenus dans ce sens : "Il ne faut pas que le concept se dilue trop ", dira l'un d'eux.

Si l'activité d'accès au droit - contrairement à l'activité judiciaire - emporte le consensus, la question de son développement, ainsi posée, soulève en réalité celle de la nature des choix fondamentaux concernant la structure dans son ensemble et du poids qui est celui, dans la décision, de l'institution judiciaire. Celle-ci, qui a su inventer un nouveau type de réponse à certains phénomènes de délinquance, est-elle prête à assumer la dimension de proximité qu'elle prétend lui conférer ? L'actuelle prédominance du judiciaire n'empêche pas l'existence d'un nombre considérable d'autres possibilités, où le « local » pourrait être plus développé, à condition qu'on le décide. La question peut se formuler en termes de compatibilité : jusqu'à quel point le dévcloppement d'une activité d'accueil véritablement ancrée dans le local, et dès lors moins exclusivement pénale ou juridique, ne vient-elle pas affaiblir l'impact d'une justice sortant - tout armée - de ses murs ? 
A. Wyvekens

Justice de proximité et proximité de la justice. Les maisons de justice et du droit

\section{Maisons de justice et proximité de la justice}

La «justice de proximité " montre ainsi ses limites. L'inscription territoriale des maisons de justice, entendue au sens d'implantation de modes spécifiques de restauration du droit dans les quartiers difficiles, se heurte en effet à la propension qu'a l'institution judiciaire à privilégier ses modes de fonctionnement propres, largement centralisateurs, et sa logique propre, plus pénale que sociale. Succédant à cette approche de l'intérieur, l'adoption d'un point de vue extérieur, sous la forme d'une lecture des représentations de la maison de justice chez les différents acteurs locaux, permet de préciser en quoi celle-ci apparait néanmoins comme le vecteur de ce qu'on appellera provisoirement un rapprochement de l'institution judiciaire. Le discours des "partenaires" locaux - municipalités participant à son financement ou autres partenaires impliqués de façon moins originaire apparaît comme le lieu d'une articulation complexe entre des préoccupations locales et centrales. Alors que le caractère limité de l'inscription territoriale des maisons de justice se reflète dans le discours de ces acteurs locaux, dont on s'aperçoit en outre qu'ils en sont, à des degrés divers, co-responsables (II.1), ce même discours se révèle porteur d'un élément nouveau de compréhension: c'est une " autre proximité » qui se fait jour (II.2).

\section{II. . La justice de proximité... tenue à distance}

Lisibles dans l'organisation et le fonctionnement du dispositif, les limites de l'inscription territoriale des maisons de justice le sont également dans le discours des acteurs locaux. La relative distance qu'ils affichent par rapport à la notion de " justice de proximité " présente deux visages : distance revendiquée chez les uns, distance acceptée chez les autres. Explicite chez des acteurs non fondateurs qui, une fois sollicités, ne négocient que prudemment leur participation, la "mise à distance ", plus diffuse rhez. les partenaires initiaux que sont les municipalités, apparaîtra chez eux, à l'analyse, comme le verso d'autres préoccupations.

\section{a. Entre curiosité et méfiance, la police et le milieu scolaire}

Partenaires seconds de la maison de justice, devenus destinataires d'interpellations fortes émanant des acteurs judiciaires - en particulier à travers les projets de saisine directe évoqués précédemment -, policiers et représentants du milieu scolaire adoptent, par rapport à cette demande, une position à dominante attentiste. Chez les uns comme chez les autres, l'activité judiciaire de la mai- 
son de justice n'est pas sans menacer des domaines de compétence considérés comme relativement réservés. Chez les policiers en outre, l'image de la maison de justice se confronte à une valorisation de la répression, propre à la profession.

Du côté des policiers, après un très consensuel "nous avons d'excellentes relations avec la maison de justice ", l'analyse de l'ensemble du propos fait apparaître, face à la maison de justice, une attitude qui va de l'approbation souriante, dépourvue d'implication forte, à un intérêt sélectif agrémenté de scepticisme et d'une certaine dose de méfiance, en passant par un bon voisinage plus axé sur la relation individuelle que sur un véritable partenariat institutionnel.

Les relations sont bonnes, sans être intensives. S'agissant de l'orientation des victimes d'infractions, l'ampleur de l'activité déployée par les acteurs policiers apparaît, comme ailleurs, liée à des dynamiques individuelles, sans que l'existence d'une maison de justice constitue en soi un élément déterminant. Quant à l'exploitation des mains courantes à des fins de traitement par la médiation, alors que les informations recueillies sur le terrain font état d'une pratique qui demeure marginale, les discours policiers sont en majorité laconiques.

Deux types de facteurs, partiellement convergents, semblent pouvoir expliquer cette distance. Mus par des préoccupations $a$ priori répressives, les policiers ont, dans un premier temps, cultivé une certaine inquiétude à l'égard de la "troisième voie " et de la " douceur " de cette justice d'un nouveau genre. Contrairement à leur crainte initiale, ils ont pu se rendre compte que la maison de justice était " autre chose que l'antichambre du classement sans suite ", que le traitement pratiqué était "d'une certaine manière répressif ». S'ils lui reconnaissent dès lors une efficacité, en particulier au niveau de la rapidité et de l'attention portée aux victimes, un fond de la méfiance initiale subsiste, qui transparaît dans leurs interrogations sur l'orientation de certaines affaires leur paraissant trop graves pour ce type de traitement - parmi elles, les outrages à agent 37 - ou sur la nécessité de ne pas multiplier les passages en maison de justice. Quant à l'exploitation des mains courantes, la réticence perçue chez les policiers invite en outre à se poser la question d'une concurrence avec le nouveau dispositif parquetier, pour une compétence que les policiers considèrent comme leur revenant naturellement. Plusieurs d'entre eux font état du rôle de médiateur que jouaient et que devraient encore pouvoir jouer les policiers localement. Ils ajoutent que leur efficacité est assurément " supérieure à celle d'un magistrat médiateur devant lequel il n'est en outre pas certain que les parties se présenteront ". La question semble ainsi se poser en termes de résistance des policiers à ce qui leur apparaît comme révélateur « d'une volonté du parquet de tout
Droit et Sociéte 33-1996 
A. Wyvekens Justice de proximité et proximité de la justice. Les maisons de justice et du droit

38. Dans ce sens, notamment, D. DAMAMME et B. JOBERT, " La politique de la ville ou l'injonction contradictoire en politique ", Revue française de science politique, 1995, p. 21-22. maîtriser "; elle serait à cet égard représentative de celle, plus générale, des enjeux de pouvoir entre police et parquet. On tiendra compte également, recoupant le premier argument, du fait que la lutte contre cette petite délinquance est moins valorisante pour les policiers, et qu'ils rechignent à effectuer ce qui leur apparait comme un travail supplémentaire dépourvu pour beaucoup de grand intérêt ${ }^{38}$.

Dans un mouvement analogue, le monde scolaire révèle quant à lui, plus nettement peut-être chez les chefs d'établissement que chez les enseignants, outre une connaissance encore floue de la structure elle-même, une méfiance persistante concernant son utilisation pour les situations problématiques survenant au sein de l'école.

L'activité proprement judiciaire de la maison de justice semble plus extérieure à l'établissement que susceptible d'en concerner directement les acteurs. Si les enseignants qui invitent le magistrat dans leur classe savent en quoi consiste le traitement judiciaire, les autres ne paraissent pas en avoir une connaissance précise. Ils le situent dans la galaxie médiation, voient la maison de justice comme un lieu de règlement des conflits, sans en connaître les modalités concrètes d'intervention. Ni les uns ni les autres, en tout cas, et cela malgré les sollicitations de certains magistrats du parquet, ne semblent faire appel facilement à ses services en matière de règlement de litiges scolaires. La possibilité de procéder à des " signalements » qui seraient traités directement par la maison de justice en dehors de toute plainte à la police, sans être catégoriquement exclue, n'est pas de pratique courante. Le discours développé à ce sujet est encore, le plus souvent, celui d'un milieu traditionnellement méfiant par rapport aux autorités policières et judiciaires, dans lequel les différends sont, dans la mesure du possible, réglés « entre soi », à l'intérieur même de l'établissement.

\section{b. L'apparente indifférence des municipalités}

La distance perceptible dans le discours des acteurs municipaux par rapport à l'idée d'une "justice de proximité » au sens défini précédemment se traduit, quant à elle, en termes de relatif désintérêt ou de méconnaissance au moins apparente de la compétence peu territorialisée de la maison de justice.

L'idée d'une action spécifique de la justice dans des territoires en difficulté, la reconnaissance de l'originalité et du caractère nécessaire de la démarche ne sont certes pas absentes des propos tenus par les élus locaux ou les membres de leur cabinet. La médiation, le souci de restauration du lien social, l'accent mis sur la citoyenneté font l'objet de remarques approbatives. On constate toutefois dans tous les sites que les détails de l'organisation et du fonctionnement semblent n'avoir qu'une importance secondaire. 
Que la maison de justice, en matiere de mediation, ne concerne

qu'une minorité de justiciables locaux, que les affaires traités ne soient pas essentiellement res affaires de voisinage cmblematiques des quartiers d'exclusion ${ }^{39}$, que la médiation ne soit, par nature, pas précisément adaptée à la délinquance des jeunes " durs " qui font d'un quartier un quartier hors droit, les acteurs municipaux n'y voient rien de rédhibitoire. Le contenu de l'action n'est pas mis en discussion. Et si l'idée d'un financement intercommunal - qui rendrait justice au profit retiré par les communes voisines resurgit régulièrement, la conviction est modérée, et le propos semble de principe.

Les représentations de la mission d'information et d'accès au droit complètent cette image. Les acteurs municipaux constatent que les habitants connaissent mal la maison de justice, à laquelle ils n'ont pas le réflexe de s'adresser. Ni cette méconnaissance, ni le hiatus entre la représentation des usagers (pour qui la maison de justice serait d'abord lieu d'accès au droit) et la leur (centrée sur le judiciaire) ne semblent toutefois constituer un sujet grave de questionnement pour les élus.

Enfin, à Vaulx-en-Velin, seule commune où la maison de justice, implantée dans la ZUP, aurait pu matérialiser une conception donnant la priorité à des missions territorialisées, la tendance du premier permanent à vouloir faire passer au second plan l'activité judiciaire, au profit d'une conception plus locale et plus sociale de la maison de justice, n'a pas suscité l'enthousiasme et s'est trouvée dissoute dans l'organisation globale du dispositif, avec l'approbation des autorités municipales.

\section{II.2. L'émergence d'une " autre proximité »}

Est-ce à dire que les acteurs locaux n'ont que faire d'une " justice de proximité " ? Que Bron, Lyon, Vaulx-en-Velin et Villeurbanne contribuent au financement des maisons de justice uniquement parce qu'elles croient naïvement, ou font semblant de croire, que la justice s'y occupe de leurs quartiers sensibles ? La réponse à ces questions peut être trouvée dans l'identification d'une autre préoccupation municipale, d'une autre attente forte, que l'institution judiciaire semble satisfaire à travers les maisons de justice.

Le discours des élus, relu à l'envers de la lecture négative qui vient d'en être faite, accrédite l'idée selon laquelle leur souci premier, qui n'est pas, en effet, celui d'un traitement judiciaire territorialisé et par là spécifique, serait en réalité celui d'un rapprochement de l'institution elle-même, dans ce qu'elle a, au contraire, de " non différent ». Les représentations identifiables chez les autres partenaires viennent, sur un mode mineur, confirmer cette hypothèse. Au-delà des réticences que leur inspire une justice différente, on discerne l'émergence d'un intérêt pour ce qui, dans le

39. Cf. les résultats du dépouillement réalisé à Lyon : la grille prévoyait un item « rapports préexistants entre les parties »: si la réponse est positive dans un peu plus de $50 \%$ des cas, moins de $8 \%$ des affaires sont des affaires de voisinage, les "rapports préexistants » étant en majorite des rapports de nature familiale ou conjugale. 
A. Wyvekens

Justice de proximité et proximité de la justice. Les maisons de justice et du droit dispositif, est pour eux l'occasion d'une plus grande familiarité avec « la " justice.

\section{a. Les villes : présence de la justice et requalification de l'espace public}

Interrogés sur les maisons de justice, les acteurs municipaux structurent leur propos autour d'une idée-force, celle du " rapprochement de la justice ». Une institution traditionnellement lointaine, avec ce que ce "lointain" comporte d'ignorance, d'incompréhension, voire de méfiance, se rapproche des citoyens. C'est la démarche elle-même, d'abord, le rapprochement en tant que tel, avant même que soit évoqué son contenu effectif, qui apparaît comme une avancée majeure.

Quant au contenu de ce rapprochement, tel que le valorisent les acteurs municipaux, il apparait plus complexe que l'image volontiers développée dans les médias d'une institution qui, en se déplaçant dans "les quartiers", viendrait y rendre une justice différente pour y réintroduire un droit disparu. La proximité à laquelle les acteurs municipaux attachent le plus de prix est d'un autre ordre. Rapprocher le judiciaire, travailler avec la justice : les attentes des municipalités se portent nettement vers la justice en tant qu'institution étatique, centrale, la justice pénale au sens fort du terme. Plusieurs éléments accréditent cette hypothèse, de manière plus ou moins nette selon les sites.

Le choix de l'implantation de trois des quatre maisons de justice, en centre-ville, à proximité de la mairie et/ou du commissariat, et non dans les quartiers les plus sensibles, apparait significatif. Ce qui est explicitement attendu, c'est avant tout une présence de la justice dans la ville au sens général du terme: "Montrer que la justice, c'est celle de tout le monde, de tous les quartiers, et pas la justice labellisée, pas quelque chose de marqué ", dira un élu. La " justice de proximité " apparait comme une justice locale en centre-ville, à la disposition de tous les habitants, service à la ville avant d'être un service destiné à certains quartiers de la ville. Cette justice-là reste avant tout la justice, qui effectue un mouvement de délocalisation, plus que de territorialisation.

S'agissant de la spécificité de l'action judiciaire des maisons de justice, c'est essentiellement l'existence et la rapidité de la réponse qui sont mises en valeur; l'accent est mis sur le traitement direct, sur le rappel à la loi, plus que sur les procédures fondées sur une approche médiatrice. Au point que la maison de justice devient pour les élus, malgré l'ćtroitesse de sa compétence locale, et comme si celle-ci n'avait que peu d'importance, " une réponse efficace qu'ils peuvent donner à leurs administrés lorsque ceux-ci se plaignent de l'insécurité »: la maison de justice, quel que soit le 
contenu de son intervention, c'est bien d'abord "la " justice qui montre qu'elle prend en compte les questions d'insécurité t).

Un autre indice est la manière dont les élus se réjouissent que la maison de justice leur permette d'entretenir une relation personnalisée avec le procureur. Avoir chez soi une maison de justice permet d'accéder facilement, sans formalités, à un chef de juridiction, à ce personnage hautement représentatif de la justice dans sa dimension répressive. "Le maire a accès au procureur... " I.a présence régulière du procureur et de certains substituts en maison de justice, la rencontre du procureur lors des comités de pilotage ont fait de ce dernier un familier des élus locaux qui peuvent à présent s'adresser à lui facilement, aussi bien pour des questions de délinquance urbaine locale que pour n'importe quels autres problèmes judiciaires. Concrètement, ce rapprochement se traduit dans les différents exemples de gestion de problèmes locaux de délinquance : ce n'est pas par la maison de justice que ceux-ci sont traités, mais grâce à elle, grâce au contact plus étroit qu'elle a instauré entre les différents acteurs locaux et l'institution, permettant dès lors une gestion plus efficace, par le pénal traditionnel, de certaines affaires locales.

Sur le plan de l'efficacité, la maison de justice est donc appréciée par les élus pour la rapidité avec laquelle les victimes de petite délinquance obtiennent réparation même si les cibles ne sont pas essentiellement locales, ainsi que pour des "effets secondaires" relatifs à la gestion par le pénal traditionnel d'affaires locales plus importantes. Elle est valorisée d'autre part en tant que vecteur d'un rapprochement plus global et multiforme entre les acteurs locaux et une justice traditionnellement vécue comme lointaine et indifférente : les occasions de rencontre dont la maison de justice est le nœud sont à cet égard particulièrement appréciées.

Au-delà du contenu instrumental, la dimension symbolique de ce rapprochement de la justice est essentielle: "Les maisons de justice sont dans la vitrine des villes. " Avoir une maison de justice, pour une ville, c'est un peu comme avoir un aéroport ou une piscine olympique. Si la présence d'un service public contribue à la requalification de l'espace public, une maison de justice, c'est en quelque sorte mieux encore, car il ne s'agit pas seulement ici de disposer d'un équipement ou d'un service, mais bien d'avoir à portée de main une parcelle de la force publique. De même qu'un quartier se trouve requalifié par la présence d'un bureau de poste annexe ou d'un commissariat, la ville qui abrite une maison de justice en tire une gratification non négligeable : avoir chez soi un peu de la justice ${ }^{41}$. L'insistance mise par d'autres villes de l'agglomération lyonnaise à tenter d'obtenir chez elles l'implantation d'une maison de justice est significative de l'importance de cet aspect. De même, les propos tenus au sujet du financement. Chacune des villes d'implantation contribue au budget des maisons de justice à
40. Illustration de la « réarticulation entre réel et symbolique » évoquée par A. GARAPON, " Justice rituelle, justice informelle, justice décentralisée ", in A. GARAPON et D. SAIAS (dir.), La justice des mineurs.

Evolution d'un modèle, Paris/Bruxelles, LGDJ/Bruylant, 1995, p. 149.

41. Sur les limites de l'approche locale des questions d'exclusion, voir M. GAUCHET, « La société d'insécurité ", in J. DONZELOT (dir.), Face à l'exclusion. Le modèle français, Paris, éd. Esprit, 1991, p. 179 et suiv. En France, " poser une question au niveau central, c'est signifier l'estime que la sociéte a de ce problème». 


\section{A. Wyvekens}

Justice de proximité et proximité de la justice. Les maisons de justice et du droit

42. "Les villes qui ont accueilli une maison de justice savent très bien que le ministère de la Justice ne serait pas apte à payer lui-mème. Les villes se disent "tant qu'on finance, ça existe et si on ne finance plus ça n'existera plus". On préfère ça que rien. " (Extrait d'entretien) 43. Sur le " binôme" magistratpolicier, voir $\mathrm{Ch}$. MOUHANNA et W. ACKERIANN, Une affaire de confiance. Les relations OPJmagistrat dans le processus pénal, Centre d'analyse, de formation et d'intervention/ IHESI/Ministère de la Justice, février 1995 (ronéo).

44. "Nous, policiers, on a le sentiment d'avoir été efficaces quand on a un mandat de dépôt. " (Extrait d'entretien)

45. On rappellera à cet égard que le créateur des maisons de justice du Val d'Oise et du Rhône instaura à Bobigny, où il fut par la suite procureur, un dispositif de traitement en temps réel non assorti de maisons de justice.

46. Cf. R. LEMAITRE, Les rapports entre la police et la justice dans le cadre de la garde à vue. L'exemple de Lyon, Montpellier, ERPC, 1993 (ronéo), p. 70, ainsi que l'article paru, sous le même titre, aux Archives de politique criminelle, n' 17, 1995, p. 67-81. L'étude montre comment « le parti pris de la confiance du parquet, nécessaire à un fonctionnement simplifié donc rapide du système, tend à modifier le rapport de contrôle classique entre magistrat et policier au profit d'une zone d'échange pour une surveillance négociée. |...] Paradoxalement, le passage rapide et obligé par le parquet, qui rend aux magistrats une place centrale dans la crise urbaine, cache une certaine perte de pouvoir dans la ielation aux policiers". concurrence d'environ $250(00) \mathrm{F}$ annuels, une somme équivalente étant ajoutée par l'État dans le cadre de la politique de la ville. Si le principe d'un financement par les villes d'une activité spécifiquement étatique n'est pas admis, et si cette situation pèse évidemment plus lourd sur les villes plus petites, les municipalités ne semblent pas pour autant prêtes à annoncer la fin de leur participation financière à l'opération ${ }^{42}$.

\section{b. La police et l'école : de la maison de justice à « la » justice}

Sans être aussi explicite, le discours que tiennent les acteurs policiers et les acteurs scolaires sur leurs relations avec la maison de justice contient néanmoins un certain nombre d'éléments de nature à renforcer l'hypothèse avancée. Si « la " médiation ne suscite chez eux qu'un enthousiasme modéré, la maison de justice présente néanmoins à leurs yeux un intérêt qui s'analyse, lui aussi, en termes de rapprochement de la justice. Le discours policier apprécie l'originalité du dispositif dans ce qu'il a de proprement pénal et centraliste, avec ses retombées en termes d'accroissement d'efficacité de la répression. Quant au discours scolaire, il montre comment la maison de justice est perçue surtout comme un lieu permettant l'accès à la justice traditionnelle.

Interrogés sur la maison de justice, tous les policiers s'attardent longuement... sur le traitement direct. Le caractère récurrent de ce détour parfois long et toujours louangeur par la description de la STD est instructif : c'est la STD, dispositif global et centralisé du traitement des affaires pénales qui est, pour les policiers, l'élément central de la représentation des maisons de justice. L'image qu'ils ont de celles-ci ne se construit pas, pour eux, autour de son inscription dans le paysage local. Le mot clé en la matière est "confiance »: le traitement direct est fondé sur un rapport de confiance entre le parquet et la police ${ }^{43}$, dont les policiers déclarent n'avoir qu'à se féliciter. Lc principal mérite du traitement en temps réel est pour eux le "retour " qu'il leur permet d'obtenir sur l'orientation donnée par le parquet aux affaires qui lui sont transmises. On notera à cet égard que leur satisfaction se mesure à l'aune de la rigueur de la solution choisie ${ }^{44}$ et que c'est l'augmentation des convocations par officier de police judiciaire, plus que le traitement en maison de justice, qui apparait ici comme un motif de satisfaction ${ }^{45}$. Au-delà d'une meilleure connaissance du sort réservé à leurs affaires, il n'est pas interdit de supposer que les policiers voient dans la " confiance " qu'implique le traitement direct l'occasion d'un accroissement de leur capacité à influer sur les orientations parquetières ${ }^{46}$. 
Pour le reste, de même que les élus ou les travailleurs sociaux, les policiers apprécient la maison de justice en tant que lieu initiateur de relations informelles, qui se concrétisent parfois en une optimisation de la collaboration entre police et parquet pour des affaires relevant, elles, de la justice répressive traditionnelle. "Confiance ", "retour ", accès aux magistrats, la maison de justice est pour les policiers, d'abord, l'occasion d'un rapprochement de l'institution dans son ensemble.

Le discours des acteurs scolaires reflète, de la même manière que les précédents, une représentation complexe de la maison de justice. Si la valorisation de l'idée de rapprochement de la justice est constante, l'essentiel des relations qui se tissent lui donne à nouveau le contenu global, non spécifique, déjà évoqué.

On a vu le caractère limité de l'intervention judiciaire de la maison de justice à propos de cas individuels ayant pour cadre le milieu scolaire. Le partenariat qui s'institue progressivement se construit pour l'essentiel sur un plan informatif, visant les élèves de manière collective, dans le cadre de l'établissement lui-même ${ }^{47}$. Les enseignants qui pratiquent ce type de collaboration l'évoquent avec enthousiasme, ceux qui projettent de l'entreprendre expriment à son propos des attentes fortes. Si leurs motivations peuvent varier, un constat est identique pour tous : l'existence d'une maison de justice leur a facilité une démarche consistant à vouloir faire mieux connaître la justice en général. La présence de représentants de la maison de justice dans les instances locales diverses, les sollicitations émanant de certains d'entre eux, l'image d'une justice moins solennelle, le voisinage géographique ont créé un rapport de proximité permettant aux enseignants de faire appel aux services d'acteurs judiciaires.

Pour la plupart des enseignants rencontrés, la motivation centrale renvoie à la problématique de la ville : leur démarche a pour objectif de réintroduire la notion de droit, l'idée de justice auprès de jeunes vivant dans un environnement où elles semblent crucllement absentes. Ce qui est attendu de la maison de justice, ici, c'est un rôle pédagogique, l'expression d'un discours fort sur le droit et la justice qui viendrait s'articuler à et renforcer «le discours tenu en permanence " par les enseignants à des élèves n'ayant pas acquis le minimum de repères dans ce domaine. Parfois, l'intervention du magistrat, destinée à un lycée " très calme, bon chic bon genre", ne répond pas à une visée pédagogique s'inscrivant dans ce registre d'une éducation civique élémentaire. L'objectif s'avère de nature technique: dans le cadre d'un cours de droit, le partenariat avec la maison de justice apparait, au même rang que n'importe quel stage en entreprise, comme un outil permettant aux élèves de se familiariser pratiquement avec le contenu théorique d'un cours. Voulant illustrer son cours de droit, l'ensei-

47. Pratiquement, le magistrat responsable d'une maison de justice, accompagné du permanent, ou le permanent seul se rend(ent) dans un établissement, où ils vont parler aux élèves de la justice en général, de la maison de justice, du métier de magistrat, du droit, de la loi. Peuvent se greffer sur cette intervention des visites effectuées par les élèves au palais de justice, où ils assistent à des audiences correctionnelles ou civiles. 
A. Wyvekens

Justice de proximité et proximité de la justice. Les maisons de justice et du droit
48. Cf., à partir d'un objet différent, les remarques plus générales formulées en termes de " politique de justice " par J. COMMAILLE, « La carte judiciaire comme projet politique", Justices, n² 2, 1995, p. 58-59. gnant trouve à la maison de justice des interlocuteurs plus abordables qu'au palais de justice, d'une part, une structure plus compréhensible pour ses élèves, d'autre part. La maison de justice est présentée alors comme l'élément ayant facilité l'accès à une institution lointaine, indépendamment de préoccupations immédiatement sécuritaires ou citoyennes.

Dans un cas comme dans l'autre, le rapprochement que la maison de justice permet d'initier va au-delà d'une connaissance de cet aspect local de la justice. Si le discours tenu aux élèves fait abondamment référence au traitement pratiqué en maison de justice, si les élèves des établissements difficiles sont encouragés à s'adresser à elle, le contenu de l'intervention la dépasse néanmoins largement. Grâce à la maison de justice, à des acteurs qui, s'y investissant, se sont trouvés sensibilisés aux préoccupations locales, c'est l'institution judiciaire tout entière qui devient plus accessible à un public de jeunes, tantôt dans un objectif explicitement " citoyen », tantôt avec une visée plus exclusivement technique ; à travers la maison de justice, c'est l'ensemble de l'institution judiciaire qui bénéficie d'une meilleure connaissance ou qui est mobilisée pour réintroduire l'idée de droit et de justice.

\section{Conclusion}

Justice de proximité. La justice avait eu du mal à entrer dans la logique partenariale de la politique de la ville, craignant de s'y compromettre et d'y perdre son identité. Elle a élaboré, avec les maisons de justice, un "produit maison " qui intègre à la fois ses préoccupations propres et celles de la politique de la ville. L'analyse de la proximité ainsi construite met en évidence la complexité - voire les contradictions - du mélange ${ }^{48}$.

S'agissant de l'activité judiciaire des maisons de justice, malgré la lecture territoriale mise à l'honneur par la politique de la ville, l'inscription de la maison de justice dans son environnement local - au sens de mise en ouvre d'un traitement adapté à la problématique de certains quartiers - se révèle limitée. La " proximité » de cette justice est autre, renvoyant à ce que sont les préoccupations et les contraintes de l'institution elle-même. Bien plus que de spatialité, la justice de proximité qu'incarnent les maisons de justice est faite d'un alliage instable de familiarité et de rapidité, dans lequel cette dernière occupe une place centrale. La proximité temporelle résume en deux mots le paradoxe d'une justice de proximité dont le contenu se construit en définitive autour d'exigences de justice dite concentrée : instauration d'une réponse effective et rapide, inspirée d'impératifs quantitatifs plus que qualitatifs et s'avérant plus répressive que ne le laisse paraître l'étiquette "médiation» qui lui reste appliquée. Au terme d'un affrontement des logiques qui voit la victoire d'une conception gestionnaire dans 
laquelle la géographie n'a qu'une importance secondaire, la proximité redeviendrait centralité, par le biais de son contenu temporel et de tout ce que celui-ci représente en termes d'efficacité de l'institution. Cette justice de proximité, où la rapidité et le souci de réponse à des situations autrefois non traitées s'adossent à l'idée d'une différence qualitative liée à des enjeux locaux, est celle du rapprochement d'une institution dont la logique propre reste dominante. La politique de la ville induirait ainsi plus une modernisation des méthodes, dans le sens d'une plus grande efficacité, qu'un réel infléchissement de l'intervention. D'un point de vue intérieur à l'institution, si ce constat est de nature à rassurer les praticiens qu'inquiète la perspective d'une " dilution » du judiciaire dans la politique de la ville ${ }^{49}$, il pose la question, tout aussi cruciale, des garanties dont ce type de traitement exige d'être assorti ${ }^{50}$. Derrière un discours d'euphémisation du pénal, n'est-ce pas, en effet, à une extension de celui-ci que l'on assiste ?

L'observation de l'activité d'accès au droit des maisons de justice prolonge ce constat. Seconde dans le temps et maintenue secondaire par un parquet qui reconnaît ne pas en faire une priorité, cette activité qui pourrait se révéler fondatrice d'une identité locale de la maison de justice n'est que peu développée: l'ensemble de la structure s'organise autour du judiciaire, comme autour du souci de maintenir l'emprise de l'institution face à tout ce qui, acteur, préoccupations, fonctionnement, pourrait la " déliter ». Même en maison de justice, c'est encore la justice qui est en scène, gardant ses objectifs particuliers, soumise à ses contraintes propres.

Proximité de la justice. Le caractère limité de l'inscription territoriale des maisons de justice - et son remplacement par une proximité dans le temps - n'apparaît pas seulement comme traduisant la prééminence de la logique de l'institution. L'enquête de terrain montre que ce type de "rapprochement " répond très largement aux préoccupations ou aux attentes des acteurs locaux. Qu'il s'agisse des élus, des policiers, des enseignants, chacun selon sa logique propre développe un discours où la maison de justice représente un mélange complexe, à dosage variable, de justice de proximité et de proximité de la justice. Pour l'école et la police, c'est le scepticisme par rapport au territorial qui apparaît d'abord, laissant ensuite émerger une valorisation de la maison de justice en tant que lieu ou occasion d'une relation plus étroite à la justice. Pour les villes, dans un équilibre inverse, le désintérêt relatif quant au contenu de l'intervention n'étonne qu'un moment ; l'essentiel ici se trouve dans la forme, avec toute la force symbolique que cela représente: les maisons de justice, en tant que présence physique de l'institution judiciaire, de la force publique en leurs murs, signe de l'intérêt, vital, que l'État porte à la communauté locale, consti-
49. Voir à ce sujet les articles de B. BRINET, J.-L. BODIGUEL et T. FIRCHow dans Droit et Société, n 26, $1994 ;$ n $^{\circ} 27,1994$ et $n^{\circ} 29,1995$.

50. Dans ce sens, notamment, F. TULKENS, La justice négociée, document de travail non publié, Département de criminologie et de droit pénal de l'UCL, Louvainla-Neuve, 1995. 
A. Wyvekens Justice de proximité et proximité de la justice. Les maisons de justice et du droit tuent sur le plan municipal un instrument de requalification de l'espace public. Pour l'ensemble des acteurs locaux, le central apparaît en définitive plus valorisé que le territorial. La maison de justice s'analyse plus en termes d'effets secondaires ou formels qu'en termes de contenu spécifique à effets directs. Ce qui compte, c'est ce à quoi elle donne accès ou ce qu'elle procure, plus qu'ellemême.

Si l'on revient aux préoccupations identitaires de l'institution judiciaire, elle peut être rassureee. La justice de proximité ne " dénature " pas la justice pénale, et cela d'autant moins que ses partenaires ne le souhaitent pas. Pour l'institution comme pour ses partenaires, ce qui apparait essentiel, c'est une justice qui se rapproche, certes, mais sans se faire forcément différente ; une justice forte qui sache se rendre plus accessible, bien plus qu'une justice dont l'infléchissement des modes d'action rendrait la présence ambiguë. Rapprocher la justice, le droit, grâce à la présence locale d'une structure dont l'activité proprement locale sera l'occasion d'un rapprochement avant d'être l'endroit d'une activité spécifique qui concernerait directement telle ou telle cible spécifique. Car la justice est apparue, au fil des années de crise urbaine, face aux limites des multiples politiques sociales ou préventives, comme un recours d'un ordre différent. Ce qui est attendu, en somme, c'est que ce soit la justice dans toute sa splendeur qui descende en ville. 